\title{
Estilo de vida e nível de atividade física de indivíduos portadores de miocardiopatia chagásica
}

\author{
Lifestyle and level of physical \\ activity in individuals with chagasic \\ myocardiopathy
}

\author{
Neila Silva Soares ${ }^{1}$ \\ Anne Karine Menezes Santos Batista ${ }^{2}$ (1) \\ Roque $\operatorname{Aras}^{3}$ (1) \\ Fernanda Warken Rosa Camelier ${ }^{4}$ (1)
}

\author{
${ }^{1}$ Autora para correspondência. Universidade do Estado da Bahia (Salvador). Bahia, Brasil. neila_soares@hotmail.com \\ 2-4Universidade do Estado da Bahia (Salvador).Bahia, Brasil. anne_kmenezes@yahoo.com.br, roque.aras@uol.com.br, fcamelier@uneb.br
}

RESUMO I INTRODUÇÃo: A doença de Chagas (DC) constitui uma infecção parasitária causada pelo protozoário flagelado Tripanosoma cruzi. Estimativas apontam a existência de, aproximadamente, cinco milhões de indivíduos infectados, principalmente na América Latina, com o Brasil datando entre 1,9 a 4,6 milhões de indivíduos sob o mesmo aspecto infeccioso. O desfecho cardíaco configura um dos aspectos mais importantes, com manifestações condizentes à disfunção ventricular sistólica ou diastólica, disfunção autonômica cardíaca e morte súbita. OBJETIVO: correlacionar o estilo de vida e o nível de atividade física de indivíduos portadores de miocardiopatia chagásica (MC). MATERIAL E MÉTODOS: Realizou-se um estudo analítico, de corte transversal, em ambulatório de referência para Miocardiopatias, com abrangência estadual. Utilizou-se formulário próprio construído pelas autoras, o qual contemplava além das variáveis clínicas e demográficas, variáveis relativas à análise do estilo de vida e atividade física dos participantes, sendo esse aplicado em sala de espera, enquanto os sujeitos aguardavam atendimento médico. RESULTADOS: Foram selecionados 74 indivíduos portadores de MC. A média de idade da amostra foi de $61,2 \pm 8,5$ anos, sendo que $50(68,0 \%)$ indivíduos eram do sexo feminino. Em relação ao nível de atividade física, houve predomínio da categoria "não ativo", correspondendo a 60 indivíduos $(71,0 \%)$. O estilo de vida foi classificado como "muito bom" para 41 (55,0\%) participantes e "bom" para $22(30,0 \%)$ participantes, não havendo indivíduos alocados na categoria "necessita melhorar" do questionário. CONCLUSÃO: Os resultados obtidos permitem concluir que, de modo geral, o estilo de vida e o nível de atividade física de indivíduos portadores de MC caracterizaram-se como "bom" / "muito bom" e não ativos, respectivamente.

PALAVRAS-CHAVE: Miocardiopatia chagásica. Estilo de vida. Atividade física.
ABSTRACT | INTRODUCTION: Chagas disease (CD) is a parasitic infection caused by the flagellated protozoan Trypanosoma cruzi. Estimates point to the existence of approximately five million infected individuals, mainly in Latin America, with Brazil dating between 1.9 and 4.6 million individuals under the same infectious aspect. The cardiac outcome is one of the most important aspects with manifestations consistent with systolic or diastolic ventricular dysfunction, cardiac autonomic dysfunction, and sudden death. OBJECTIVE: To correlate the lifestyle and physical activity level of individuals with Chagas cardiomyopathy (CM). MATERIAL AND METHODS: A cross-sectional analytical study was carried out in a reference outpatient clinic for cardiomyopathies, with state coverage. A form was created by the authors, which included, in addition to the clinical variables and demographic, variables related to the analysis of the participants' lifestyle and physical activity, this being applied in the waiting room, while the subjects waited for medical care. RESULTS: Seventy-four individuals with CM were selected. The mean age of the sample was $61.2 \pm 8.5$ years, with $50(68.0 \%)$ individuals being female. Regarding the level of physical activity, the "not active" category was predominant, corresponding to 60 individuals (71.0\%). The lifestyle was classified as "very good" for 41 (55.0\%) participants and "good" for 22 (30.0\%) participants, with no individuals allocated to the "need to improve" category of the questionnaire. CONCLUSION: The results obtained allow us to conclude that, in general, the lifestyle and the level of physical activity of individuals with CM were characterized as "good" / "very good" and not active, respectively.

KEYWORDS: Chagasic cardiomyopathy. Lifestyle. Physical activity. 


\section{Introdução}

A doença de Chagas (DC) constitui uma infecção parasitária causada pelo protozoário flagelado Tripanosoma cruzi 1-3. . Descoberta em 1909 pelo médico sanitarista Carlos Chagas, a despeito das reduções observadas na incidência ao longo dos anos ${ }^{1-2}$, estimativas apontam a existência de, aproximadamente, cinco milhões de indivíduos infectados, principalmente na América Latina ${ }^{3}$, com o Brasil datando entre 1,9 a 4,6 milhões de indivíduos sob o mesmo aspecto infeccioso, o que representa 1 a $2,4 \%$ da população ${ }^{3,4}$.

Países do Cone Sul, em 1995, comprometeram-se em buscar a eliminação do vetor responsável pela transmissão dessa enfermidade ${ }^{5}$. Dessa forma, países como o Uruguai, em 1997, e o Chile, em 1999, foram certificados inicialmente, sendo o Brasil mais tardiamente reconhecido como Estado cuja transmissão do vetor fora erradicada ${ }^{5}$. Ademais, em 2018, importante decisão fora tomada no âmbito do reconhecimento da importância dessa doença no país, a qual, apesar da redução na incidência, possui uma morbidade que impacta a vida de milhões de brasileiros ${ }^{6}$. A aprovação do Protocolo Clínico e Diretrizes Terapêuticas no âmbito do SUS representou o reconhecimento de uma enfermidade clinicamente negligenciada, apesar do custo social trazido ao sistema ${ }^{5,6}$.

A enfermidade pode apresentar um caráter sistêmico de acometimento, conduzindo, em sua fase crônica, a alterações em órgãos-alvo como coração - compondo a forma cardíaca - esôfago e cólon - compondo a forma digestiva $a^{3,4}$. O desfecho cardíaco configura um dos aspectos mais importantes, respondendo por 25 a 30\% dos casos crônicos, com manifestações condizentes à disfunção ventricular sistólica ou diastólica, arritmias, disfunção autonômica cardíaca, tromboembolia e morte súbita ${ }^{4}$.

Como outras doenças crônicas, a miocardiopatia chagásica (MC) sofre influência de fatores relativos ao estilo de vida, traduzido por padrões de comportamentos identificáveis, resultantes da interação do ambiente socioeconômico, dos valores culturais e das atitudes pessoais do indivíduo ${ }^{8,9}$. De modo semelhante, as limitações funcionais provenientes da doença, principalmente nas fases mais avançadas da insuficiência cardíaca (IC), em decorrência de sintomas como palpitações, dor precordial e dispneia, interferem no estilo de vida, havendo evidência favorável quanto à redução da produtividade e alteração do equilíbrio dos componentes que geram bem-estar ${ }^{8,9}=$.

Nesse contexto, insere-se o exercício físico como alterativa favorável à melhora da capacidade funcional desses indivíduos ${ }^{7-9}$. Utilizando-se do Questionário de Perfil de Atividade Humana, Costa et al., em 2019, avaliaram a associação entre essa ferramenta, a capacidade funcional, a classe funcional e função sistólica em indivíduos com CC7 Sexo, idade mais avançada, classe funcional (NYHA II e III), pressão sistólica e diastólica, índice de massa corporal e o baixo consumo de oxigênio foram associados com menores valores obtidos no questionário, demonstrando o impacto da doença sobre a capacidade para realização de atividades de vida diária (AVDs) por esses indivíduos ${ }^{7}$.

Considerando que a DC permanece negligenciada pelas organizações de saúde, no Brasil e no mundo, dificultando o diagnóstico precoce, bem como a necessidade de identificar fatores que interferem nas condições de saúde apresentadas por essa população, o objetivo do presente estudo foi caracterizar o estilo de vida e o nível de atividade física de indivíduos portadores de miocardiopatia chagásica (MC).

\section{Material e métodos}

Realizou-se um estudo analítico, de corte transversal, em ambulatório de referência para Miocardiopatias, com indivíduos portadores de MC confirmada em prontuário por teste de sorologia positivo para Doença de Chagas, que compareceram a consulta médica previamente agendada, acima de 18 anos e de ambos os sexos. Dificuldades de comunicação que comprometessem o entendimento do instrumento bem como, a limitação quanto ao acesso a prontuários, constituíram-se como critérios de exclusão da pesquisa; com os dados primários coletados por meio de formulário aplicado e os secundários obtidos em prontuários. 
A coleta de dados ocorreu no período de fevereiro de 2014 a junho de 2014, precedida em uma sala de espera do referido ambulatório, enquanto os indivíduos aguardavam a consulta com o médico de referência. Para a obtenção dos dados sociodemográficos e clínicos, utilizou-se um formulário de autoria própria, aplicado uma vez, no momento da espera da consulta, por pesquisador único e de forma clara, de modo a assegurar a padronização do processo de coleta e para que as dúvidas sobre quaisquer perguntas fossem sanadas. Em seguida, aplicou-se o questionário referente aos quesitos sobre o estilo de vida dos participantes, sendo os mesmos caracterizados conforme pontuação às perguntas.

Para avaliação do estilo de vida dos indivíduos, usouse o questionário validado no Brasil para adultos jovens, "Estilo de vida FANTASTICO"11, desenvolvido em 1984, no Canadá, com a finalidade de auxiliar médicos que trabalham na atenção primária ${ }^{11}$. Constituído por vinte e cinco questões fechadas, dispostas na forma de escala do tipo Likert, o mesmo explora nove domínios sobre os componentes físicos, psicológicos e sociais do estilo de vida dos indivíduos no último mês ${ }^{10,11}$.

As variáveis utilizadas originaram-se do acrômio do termo FANTASTICO, em sua correspondente forma em inglês - FANTASTIC, referentes aos domínios contemplados pelo instrumento: família e amigos; atividade física; nutrição; tabaco e tóxicos; álcool; sono, cinto de segurança, estresse e sexo seguro; tipo de comportamento; introspecção e trabalho. A pontuação total varia de zero a cem, o que permite classificar o estilo de vida em: excelente (85-100 pontos); muito bom (70 a 84 pontos); bom (55 a 69 pontos); regular (35 a 54 pontos); e necessita melhorar (0 a 34 pontos $)^{10,11}$.

A atividade física foi mensurada por meio do Questionário Internacional de Atividade Física (IPAQ), em sua versão curta, composta de sete questões abertas, permitindo mensurar o tempo gasto pelo indivíduo, na realização de atividades de diferentes dimensões (caminhadas e esforços físicos de intensidade moderada e vigorosa), bem como durante a inatividade física (posição sentada), durante a semana12,13. Dessa forma, para ser classificado como ativo o indivíduo deverá ter realizado atividade vigorosa $\geq$ três dias na semana e $\geq 20$ minutos por sessão; ou atividade moderada ou caminhada $\geq$ cinco dias na semana e $\geq 30$ minutos por sessão; ou qualquer atividade somada, $\geq$ cinco dias e $\geq 150$ minutos na semana (caminhada + atividade moderada + atividade vigorosa). Indivíduos que realizaram atividade física, porém não o suficiente para ser classificado como ativo, ou que não cumpriram dez minutos contínuos de atividade física, na semana anterior, foram categorizados como não ativos ${ }^{12-13}$.

As variáveis demográficas avaliadas incluíram a idade, expressa em anos, o sexo, dicotomizado em feminino ou masculino, e a naturalidade, classificada em soteropolitano, proveniente do interior da Bahia ou de outro estado. A escolaridade foi categorizada em baixa, para os indivíduos que não concluíram o ensino fundamental ou segundo grau do ensino médio, média, para os que concluíram o segundo grau do ensino médio, e alta, para aqueles que cursaram ou estavam cursando o ensino superior. A cor da pele foi dicotomizada em negro e não negro, e o relacionamento conjugal, categorizado por sim ou não.

As variáveis socioeconômicas constituíram-se em renda, classificada por menor que um salário mínimo, entre 1-2 salários mínimos, entre 3-4 salários mínimos, entre 4-5 salários mínimos, e maior que cinco salários mínimos; e jornada de trabalho, dicotomizada em sim ou não. As variáveis clínicas corresponderam ao peso, expresso em Kg; a altura, expressa em metros (m) e o índice de massa corporal (IMC), expresso em $\mathrm{Kg} / \mathrm{m} 2$. A classe funcional de Insuficiência Cardíaca (IC), de acordo com a New York Heart Association (NYHA), foi subdividida em classes I-II/III e a fração de ejeção do ventrículo esquerdo (FEVE), expressa em (\%). Categorizou-se a presença de comorbidades por sim ou não, assim como o uso de marcapasso, sendo o tempo de descoberta da doença definido em anos a partir do diagnóstico inicial. 
No presente estudo, a amostra fora obtida em caráter de conveniência, conforme a ida dos indivíduos ao ambulatório de Miocardiopatia Chagásica. O banco de dados foi estruturado no programa computacional Microsoft Excel (XP) e a análise de dados pelo Statistical Package for the Social Sciences (SPSS) versão 17, com os valores apresentados em média, desvio-padrão e proporções, quando indicado. Realizou-se a análise de curtose para identificar o comportamento das variáveis continuas em relação à normalidade. O teste t-student foi utilizado para a comparação da média da pontuação total obtida no questionário "estilo de vida FANTASTICO" em relação à distribuição por sexo (masculino/feminino), classe funcional (I-II/III), escolaridade (baixa/média/alta), cor (negros/ não negros) e nível de atividade física (ativo/não ativo) e o teste qui-quadrado, para avaliar a associação entre a pontuação dos questionários de estilo de vida e atividade física com a gravidade da doença. Um p<0,05 foi considerado estatisticamente significante.

Procurou-se garantir o anonimato e a confidencialidade das informações obtidas, e o consentimento para a participação e a autonomia no estudo obteve-se por meio da aplicação do Termo de Consentimento Livre e Esclarecido emitido em duas cópias, assinado conforme orientação da Resolução 466/12 do CNS. O projeto de pesquisa foi aprovado pelo Comitê de Ética em Pesquisa da Universidade do Estado da Bahia, sob parecer 532.517/2013, e do Hospital Universitário Professor Edgard Santos/UFBA, parecer n. 624.701/2014 (CAAE 20188413.5.3001.0049).

\section{Resultados}

Foram selecionados 90 indivíduos portadores de $\mathrm{MC}$ em ambulatório de referência, localizado na cidade de Salvador, Bahia. Conforme critérios de inclusão previamente citados, 74 indivíduos compuseram a amostra, com dezesseis exclusões $(17,7 \%)$ relativas à limitação ao acesso de prontuários. A média de idade da amostra foi de $61,2 \pm 8,5$ anos, sendo que 50 (68\%) indivíduos eram do sexo feminino; as outras características sociodemográficas estão descritas na Tabela 1. 
Tabela 1. Características sociodemográficas dos indivíduos portadores de miocardiopatia Chagásica atendidos em ambulatório de referência, Salvador, Bahia- 2014

\begin{tabular}{|c|c|c|}
\hline Variáveis & $n=74$ & $\%$ \\
\hline \multicolumn{3}{|l|}{ Sexo } \\
\hline Feminino & 50 & 68,0 \\
\hline Masculino & 24 & 32,0 \\
\hline \multicolumn{3}{|l|}{ Cor } \\
\hline Negros & 51 & 69,0 \\
\hline Não negros & 23 & 31,0 \\
\hline \multicolumn{3}{|l|}{ Religião } \\
\hline $\operatorname{Sim}$ & 73 & 99,0 \\
\hline Não & 1 & 1,0 \\
\hline \multicolumn{3}{|l|}{ Escolaridade } \\
\hline Baixa & 62 & 84,0 \\
\hline Média & 12 & 16,0 \\
\hline \multicolumn{3}{|l|}{ Situação conjugal } \\
\hline $\operatorname{sim}$ & 37 & 50,0 \\
\hline Não & 37 & 50,0 \\
\hline \multicolumn{3}{|c|}{ Renda (em salários mínimos) } \\
\hline$<1$ & 6 & 6,0 \\
\hline $1-2$ & 68 & 64,0 \\
\hline $2-3$ & 6 & 6,0 \\
\hline \multicolumn{3}{|l|}{ Atividade Laboral } \\
\hline Sim & 15 & 20,0 \\
\hline Não & 59 & 80,0 \\
\hline \multicolumn{3}{|l|}{ Naturalidade } \\
\hline Soteropolitano & 6 & 8,1 \\
\hline Interior da Bahia & 66 & 89,2 \\
\hline Outro estado & 2 & 2,7 \\
\hline Variáveis & Média & Desvio-padrão \\
\hline Idade (anos) & 61,2 & 8,5 \\
\hline
\end{tabular}

Quanto aos aspectos clínicos avaliados, presentes na Tabela 2, observou-se que 71 (96,0\%) indivíduos apresentaram-se entre as classes funcionais I-II da NYHA e possuíam FEVE média de 52,2 $\pm 16,0 \%$. Em relação ao nível de atividade física, houve predomínio da categoria "não ativo", correspondendo a 60 indivíduos (81\%). O estilo de vida foi classificado como "muito bom" para 41 (55\%) participantes e "bom" para 22 (30\%) participantes, não havendo indivíduos alocados na categoria "necessita melhorar" do questionário. 
Tabela 2. Frequência dos parâmetros clínicos de indivíduos portadores de miocardiopatia Chagásica atendidos em Ambulatório de referência, Salvador, Bahia. 2014

\begin{tabular}{|c|c|c|}
\hline Variáveis & $n=74$ & $\%$ \\
\hline \multicolumn{3}{|l|}{ NYHA } \\
\hline Classe I-II & 71 & 96,0 \\
\hline Classe III & 3 & 4,0 \\
\hline \multicolumn{3}{|l|}{ Marca-passo } \\
\hline Sim & 14 & 19,0 \\
\hline Não & 60 & 81,0 \\
\hline \multicolumn{3}{|l|}{ Comorbidades } \\
\hline Sim & 62 & 84,0 \\
\hline Não & 12 & 16,0 \\
\hline \multicolumn{3}{|l|}{ FANTASTICO } \\
\hline Regular & 2 & 3,0 \\
\hline Bom & 22 & 30,0 \\
\hline Muito bom & 41 & 55,0 \\
\hline Excelente & 9 & 12,0 \\
\hline \multicolumn{3}{|l|}{ IPAQ- versão curta } \\
\hline Sedentário & 16 & 22,0 \\
\hline Irregularmente ativo & 44 & 59,0 \\
\hline \multicolumn{3}{|l|}{ Ativo } \\
\hline & 14 & 19,0 \\
\hline Parâmetros quantitativos & Média & Desvio-Padrão \\
\hline IMC $\left(\mathrm{Kg} / \mathrm{m}^{2}\right)$ & 26,3 & 5,3 \\
\hline FEVE (\%) & 52,2 & 16,0 \\
\hline Tempo da descoberta da doença (anos) & Mediana & $\begin{array}{c}\text { Intervalo } \\
\text { interquartil }\end{array}$ \\
\hline & 18 & $51(2-53)$ \\
\hline
\end{tabular}

A média da pontuação alcançada no FANTASTICO correspondeu a 73,2 $\pm 8,8$. Os domínios com menor escore corresponderam a "atividade física" e "sono, segurança, estresse e sexo seguro", 2,2 $\pm 1,8$ e 12,0 \pm 4,0, respectivamente (Tabela 3).

Tabela 3. Descrição dos valores obtidos em cada domínio do questionário estilo de vida FANTASTICO, dos indivíduos portadores de miocardiopatia chagásica atendidos em ambulatório de referência, Salvador, Bahia - 2014

\begin{tabular}{|c|c|c|c|}
\hline \multirow[t]{2}{*}{ Domínios } & \multicolumn{2}{|c|}{ Pontuação FANTASTICO } & \multirow{2}{*}{$\begin{array}{l}\text { PREVISTO } \\
\text { Mín-Máx }\end{array}$} \\
\hline & Média (DP)* & Mín-Máx & \\
\hline Família e Amigos & $6,5 \pm 2,1$ & $1-8$ & $0-8$ \\
\hline Atividade Física & $2,2 \pm 1,8$ & $0-4$ & $0-8$ \\
\hline Nutrição & $9,0 \pm 2,5$ & $3-12$ & $0-12$ \\
\hline Tabaco e Tóxicos & $14,1 \pm 1,6$ & $9-16$ & $0-16$ \\
\hline Álcool & $11,7 \pm 1,1$ & $6-12$ & $0-12$ \\
\hline Sono, segurança, estresse e sexo seguro & $12,0 \pm 4,0$ & $0-20$ & $0-20$ \\
\hline Tipo de comportamento & $5,2 \pm 2,3$ & $0-8$ & $0-8$ \\
\hline Introspecção & $9,0 \pm 2,5$ & $2-12$ & $0-12$ \\
\hline Trabalho & $3,7 \pm 0,9$ & $0-4$ & $0-4$ \\
\hline FANTÁSTICO total & $73,2 \pm 8,8$ & $52-93$ & $0-100$ \\
\hline
\end{tabular}

*DP=desvio-padrão/*^Mín-Máx=valor 
Não houve diferença estatística entre a pontuação total nos diferentes domínios do estilo de vida em relação à distribuição por sexo $(p=0,33)$, cor da pele $(p=0,14)$ e classe funcional $(p=0,91)$. Quando associado com o relacionamento conjugal dos participantes, obteve-se resultado estatisticamente significativo $(p=0,01)$ (Tabela 4).

Tabela 4. Correlação entre a classificação obtida no questionário estilo de vida FANTASTICO com variáveis clínicas e demográficas dos indivíduos portadores de miocardiopatia chagásica atendidos em ambulatório de referência, Salvador, Bahia-2014

\begin{tabular}{|c|c|c|c|c|c|c|c|c|c|}
\hline \multirow[t]{3}{*}{ Variáveis } & \multicolumn{8}{|c|}{ Classificação FANTÁSTICO } & \multirow{3}{*}{ Valor de $p$} \\
\hline & \multicolumn{2}{|c|}{ Regular } & \multicolumn{2}{|c|}{ Bom } & \multicolumn{2}{|c|}{ Muito bom } & \multicolumn{2}{|c|}{ Excelente } & \\
\hline & $\mathbf{n}$ & $\%$ & $\mathbf{n}$ & $\%$ & $\mathbf{n}$ & $\%$ & $\mathbf{n}$ & $\%$ & \\
\hline \multicolumn{10}{|l|}{ Sexo } \\
\hline Feminino & 2 & 4,0 & 16 & 32,0 & 28 & 56,0 & 4 & 8,0 & 0,33 \\
\hline Masculino & & & 6 & 25,0 & 13 & 54,2 & 5 & 20,8 & \\
\hline \multicolumn{10}{|l|}{ Escolaridade } \\
\hline Baixa & 2 & 3,0 & 17 & 27,0 & 34 & 55,0 & 9 & 15,0 & 0,41 \\
\hline Média & - & - & 5 & 41,7 & 7 & 58,3 & - & - & \\
\hline \multicolumn{10}{|l|}{ Cor } \\
\hline Negro & 2 & 4,0 & 12 & 23,5 & 32 & 62,7 & 5 & 9,8 & 0,14 \\
\hline Não negro & - & - & 10 & 43,5 & 9 & 39,1 & 4 & 17,4 & \\
\hline \multicolumn{10}{|l|}{ Situação conjugal } \\
\hline $\operatorname{Sim}$ & - & - & 7 & 18,9 & 22 & 59,5 & 8 & 21,6 & 0,01 \\
\hline Não & 2 & 5,4 & 15 & 40,5 & 19 & 51,4 & 1 & 2,7 & \\
\hline \multicolumn{10}{|l|}{ NYHA* } \\
\hline Classe I-II & 2 & 2,8 & 21 & 29,6 & 39 & 54,9 & 9 & 12,7 & 0,91 \\
\hline Classe III & - & - & 1 & 33,3 & 2 & 66,7 & - & - & \\
\hline \multicolumn{10}{|l|}{ Comorbidades } \\
\hline Sim & 2 & 3,3 & 19 & 30,6 & 32 & 51,6 & 9 & 14,5 & 0,36 \\
\hline Não & - & - & 3 & 25,0 & 9 & 75,0 & - & - & \\
\hline
\end{tabular}

* New York Heart Association

O nível de atividade física não apresentou diferença estatística quando relacionado com a distribuição por sexo $(p=0,67)$, classificação do FANTASTICO $(p=0,72)$ e classe funcional $(p=0,47)$, (Tabela 5$)$. 
Tabela 5. Associações entre categorias do IPAQ com o sexo e os parâmetros clínicos de indivíduos portadores de miocardiopatia chagásica, atendidos em ambulatório de referência, Salvador, Bahia-2014

\begin{tabular}{|c|c|c|c|c|c|c|c|}
\hline \multirow{3}{*}{$\begin{array}{l}\text { Variáveis } \\
\text { Sexo }\end{array}$} & \multicolumn{6}{|c|}{ Nível de atividade física - IPAQ* } & \multirow[t]{3}{*}{ Valor de $p$} \\
\hline & \multicolumn{2}{|c|}{$\mathbf{S}^{\star \star}$} & \multicolumn{2}{|c|}{$I A^{\star \star \star}$} & \multicolumn{2}{|c|}{$A^{\star \star \star \star}$} & \\
\hline & $\mathbf{n}$ & $\%$ & $\mathbf{n}$ & $\%$ & $\mathbf{n}$ & $\%$ & \\
\hline Feminino & 10 & 20,0 & 31 & 62,0 & 9 & 18,0 & 0,81 \\
\hline Masculino & 6 & 25,0 & 13 & 54,2 & 5 & 20,8 & \\
\hline \multicolumn{8}{|l|}{ FANTÁSTICO } \\
\hline Regular & 1 & 50,0 & 1 & 50,0 & - & - & \\
\hline Bom & 5 & 22,8 & 14 & 63,6 & 3 & 13,6 & 0,60 \\
\hline Muito bom & 10 & 24,4 & 22 & 53,6 & 9 & 22,0 & \\
\hline Excelente & - & - & 7 & 77,8 & 2 & 22,2 & \\
\hline \multicolumn{8}{|l|}{ NYHA ${ }^{\star \star \star \star}$} \\
\hline Classe I-II & 15 & 21,1 & 43 & 60,6 & 13 & 18,3 & 0,64 \\
\hline Classe III & 1 & 33,3 & 1 & 33,3 & 1 & 33,4 & \\
\hline \multicolumn{8}{|l|}{ Comorbidades } \\
\hline Sim & 14 & 22,5 & 35 & 56,5 & 13 & 21 & 0,45 \\
\hline Não & 2 & 16,7 & 9 & 75,0 & 1 & 8,3 & \\
\hline \multicolumn{8}{|l|}{ Marca-passo } \\
\hline $\operatorname{Sim}$ & 4 & 28,6 & 7 & 50,0 & 3 & 21,4 & 0,70 \\
\hline Não & 12 & 20,0 & 37 & 61,7 & 9 & 18,3 & \\
\hline \multicolumn{8}{|l|}{ Tempo de descoberta } \\
\hline$\leq 10$ anos & 9 & 42,9 & 8 & 38,1 & 4 & 19,0 & 0,01 \\
\hline$>10$ anos & 7 & 13,2 & 36 & 67,9 & 10 & 18,9 & \\
\hline
\end{tabular}

\section{Discussão}

No presente estudo, observou-se que o estilo de vida e o nível de atividade física de indivíduos portadores de MC caracterizaram-se como "bom" / "muito bom" e "não ativos", respectivamente. Apesar de estudos variados apontarem para o desfecho mais negativo de sujeitos com IC de etiologia chagásica14,15, quando comparado com outras etiologias, os hábitos de vida categorizados como "bom" / "muito bom" poderiam estar relacionados à presença de variáveis clínicas, como FEVE e classe funcional, com menor grau de alteração, equiparado aos outros estudos ${ }^{14,15}$.

O conhecimento do estilo de vida tem sido utilizado como ferramenta de suporte para prática profissional em algumas populações-alvo ${ }^{16-18}$. Elementos chaves em sua caracterização constituem-se como fatores essenciais na descrição das condições de saúde de grupos específicos ${ }^{16-18}$. Araújo et al. ${ }^{17}$, em 2011 , determinaram o estilo de vida de indivíduos hipertensos, provenientes do município de Piraí, Rio de Janeiro, obtendo-se resultado, em geral, "muito bom". Tais autores associaram às prováveis modificações realizadas por esses sujeitos, tais como, adoção de uma dieta balanceada, realização de atividade física e diminuição do consumo de álcool, possível explicação para o desfecho encontrado.

No geral, a pontuação obtida no FANTASTICO pelos indivíduos da presente amostra foi elevada corroborando com o estudo de Moctezuma et al. ${ }^{16}$, em 2003, realizado com sujeitos diabéticos, o qual apresentou escore médio de 71 pontos. Na análise dos domínios do instrumento FANTASTICO, observou-se pior desempenho nas categorias "atividade física", ratificada pelos resultados obtidos com o IPAQ, e "sono, segurança, estresse e sexo seguro".

A qualidade do sono em indivíduos com IC tem sido alvo de discussões mais recentes, em virtude do impacto que esse apresenta sobre a capacidade funcional do indivíduo ${ }^{19,20}$. Bornhausen, Kessler \& Gasperin, em 2018, em estudo realizado com 43 indivíduos cardiopatas isquêmicos no município de Itajaí, utilizando o questionário Pittsburg Sleep Quality Index, obtiveram proporção de $88,3 \%$ de sujeitos que relataram distúrbios do sono. Dentre as principais queixas, as dificuldades de iniciar o sono, os múltiplos despertares noturnos, o despertar precoce e 
a sonolência/fadiga persistente durante o dia, constituíram os sintomas mais importantes dentre os destacados pelas autoras ${ }^{19}$.

De modo adicional, utilizando-se do mesmo questionário, Edmealem et al., em 2020, no hospital de Debre Markos, avaliaram a qualidade do sono de 384 indivíduos portadores de doenças crônicas, Diabetes Mellitus, Hipertensão Arterial e Insuficiência Cardíaca, no período entre fevereiro a abril de 2018. Cerca de $75 \%$ da amostra referiu distúrbios do sono, sendo a idade, a escolaridade e a percepção do prognóstico da doença um dos fatores associados a pior qualidade do sono dentre os participantes ${ }^{20}$.

Apesar de a classificação geral obtida no FANTASTICO ter indicado estilo de vida "bom" / "muito bom", ressalta-se que o questionário quantifica a informação referente a percepção do sujeito em relação ao seu modo de vida, sendo esse, portanto, de caráter subjetivo e dependente das circunstâncias ocorridas no determinado período de coleta de dados. O fato de ter sido delineado como uma ferramenta útil para avaliação dos hábitos de vida de indivíduos atendidos em unidades de atenção primária ${ }^{13}$ transforma o questionário em uma ferramenta importante ao conferir, aos profissionais, de forma rápida e fácil, uma visão geral das condições de saúde do indivíduo. No entanto, a não inclusão de parâmetros condizentes com a MC, tais como, tempo de diagnóstico, aderência terapêutica, classe funcional da IC, comorbidades associadas, reduzem a acurácia do instrumento na predição do estilo de vida nessa população.

A maioria dos sujeitos da presente amostra não realizava atividade física regular, dado corroborado pelo estudo de Jackson et al. ${ }^{21}$, em 2012, ao analisar a presença de fatores metabólicos, de saúde mental e socioeconômicos sobre migrantes latino-americanos com DC, acompanhados em hospital universitário de Geneva, Suíça. Esse estudo obteve proporção de $62,8 \%$ de indivíduos com atividade física irregular, atribuindo tal resultado às mudanças de comportamento observadas entre esses após o processo migratório.

Mais recentemente, o ensaio HF-ACTION, de 2019, avaliou a capacidade de exercício de 1494 sujeitos com Insuficiência cardíaca sistólica crônica, utilizando para tal, a versão curta do IPAQ22. Observou-se que aqueles indivíduos categorizados no tercil mais alto de atividade física apresentaram consumo maior de oxigênio pico (VO2 pico), maior duração no teste de exercício cardiopulmonar (TCP) e maior distância no teste de caminhada de seis minutos (TC6) quando comparado aos outros tercis, demonstrando que indivíduos cardiopatas, fisicamente mais ativos, apresentavam um perfil clinico mais favorável e maior capacidade de exercício quando comparado aqueles com menor nível de atividade física를.

O estilo de vida correlacionou-se, de modo significativo, com a existência de relacionamento conjugal entre os sujeitos da amostra, dado ratificado por outros estudos ${ }^{23,24}$ que demonstraram a influência positiva exercida pelo suporte social sobre os hábitos de vida de indivíduos predominantemente mais velhos. Serafim et al. ${ }^{23}$, em 2010, com população hipertensa, ressaltaram que, dentre os fatores que poderiam influenciar o tratamento e, consequentemente, o controle da hipertensão, o estado civil desses sujeitos configurou importante variável biossocial.

A presença de um companheiro, como parceiro social mais frequente, apresenta relevante importância em consequência do suporte fornecido, em estudo com idosas ${ }^{23,24}$, ao prover troca permanente de assistência entre os membros, o que contribui para estilos de vida mais saudáveis. Urzúa 24 , em 2008, com amostra de sujeitos chilenos com doenças crônicas diversas, observou que, dentre as variáveis que influenciam a qualidade de vida desses indivíduos, o apoio social, apesar da ausência de correlação significativa, mostrou-se importante na medida em que o reconhecimento da existência de apoio, mais que a presença real desse apoio esteve relacionada à melhor qualidade de vida. Dessa forma, o suporte social fornecido pela presença de um relacionamento conjugal justificaria o resultado encontrado.

A avaliação dos hábitos de vida apresentados por esses participantes faz-se necessária na medida em que fornece aos profissionais, de modo rápido e fácil, informações valiosas referentes a parâmetros funcionais do indivíduo, permitindo a obtenção de dados relativos às condições de saúde desse grupo específico. Novos estudos, no entanto, que avaliem o impacto causado pelas alterações no estilo de vida consequentes à evolução da IC de etiologia chagásica é necessária de modo a complementar o conhecimento concernente a esses indivíduos possibilitando a melhoria das ações educativas e terapêuticas existentes. 
Novos estudos, no entanto, que avaliem o impacto causado pelas alterações no estilo de vida consequentes à evolução da IC de etiologia chagásica, são necessários, de modo a complementar o conhecimento concernente a esses indivíduos, possibilitando a melhoria das ações educativas e terapêuticas existentes

O estudo apresentou algumas limitações. Primeiro, o reduzido número de publicações provenientes de grupos de pesquisa de outras nacionalidades, apesar de configurar aspecto relevante da produção de conhecimento por brasileiros, dificultou a comparação com dados internacionais. Segundo, ao fato de ter sido realizado em um único centro para o tratamento de indivíduos com MC, não permitiram criar generalizações na análise realizada, sendo esses resultados avaliados de modo parcimonioso.

\section{Conclusão}

Os resultados obtidos permitem concluir que, de modo geral, o estilo de vida parece relacionar-se com o nível de atividade física de indivíduos portadores de MC sendo esses caracterizados como "bom" / "muito bom" e não ativos, respectivamente. A avaliação dos hábitos de vida apresentados por esses participantes faz-se necessário na medida em que fornece aos profissionais, de modo rápido e fácil, informações valiosas referentes a parâmetros funcionais do indivíduo, permitindo a obtenção de dados relativos às condições de saúde desse grupo específico.

\section{Contribuições dos autores}

Soares NS participou da concepção, delineamento, coleta de dados, busca e análise estatística dos dados de pesquisa, interpretação dos resultados e redação do artigo científico. Batista AKM participou da interpretação dos resultados e redação do artigo científico. Aras R participou da interpretação dos resultados. Camelier FWR participou da concepção, delineamento, coleta de dados, busca e análise estatística dos dados de pesquisa, interpretação dos resultados e redação do artigo científico.

\section{Conflitos de interesses}

Nenhum conflito financeiro, legal ou político envolvendo terceiros (governo, empresas e fundações privadas, etc.) foi declarado para nenhum aspecto do trabalho submetido (incluindo, mas não se limitando a subvenções e financiamentos, participação em conselho consultivo, desenho de estudo, preparação de manuscrito, análise estatística, etc.).

\section{Referências}

1. Andrade DV, Gollob KJ, Dutra WO. Acute chagas disease: new global challenges for an old neglected disease. PLoS Negl Trop Dis. 2014;8(7):e3010. https://doi.org/10.1371/journal. pntd.0003010

2. Andrade JA, Marin-Neto JA, Paola AAV, Vilas-Boas F, Oliveira GMM, Bacal F, et al. Sociedade Brasileira de Cardiologia. I Diretriz Latino Americana para o Diagnóstico e Tratamento da Cardiopatia Chagásica. Arq Bras Cardiol. 2011;96(6):434-42. https://doi. org/10.1590/s0066-782X2011000600002

\section{Pérez-Molina JA, Molina I. Chagas Disease. Lancet. 2018;391(10115):82-94. https://doi.org/10.1016/S0140- $\underline{6736(17) 31612-4}$}

4. Costa HS, Lima MMO, Costa FSM, Chaves AT, Nunes MCP, Figueiredo PHS, et al. Reduced functional capacity in patients with Chagas disease: a systematic review with meta-analysis. Rev. Soc. Bras. Med. Trop. 2018;51(4):421-6. https://doi.org/10.1590/00378682-0158-2018.18

5. Ramos ANJ, Carvalho DM. Os diferentes significados da certificação conferida ao Brasil como estando livre da doença de Chagas. Cad. Saúde Pública. 2001;17(6):1403-12. https://doi. org/10.1590/S0102-311X2001000600011

6. Portaria $n^{\circ}$ 57, de 30 de outubro de 2018 (Brasil). Torna pública a decisão de aprovar o Protocolo Clínico e Diretrizes Terapêuticas da doença de Chagas, no âmbito do Sistema Único de Saúde SUS. [Internet]. Ministério da saúde; 2018.

Disponível em: http://conitec.gov.br/images/PCDT_Doenca_de_ Chagas.pdf

7. Costa HS, Lima MMO, Vieira CFD, Silva WT, Nunes MDCP, Rocha MOC, et al. Assessment of functional performance in Chagas heart disease by Human Activity Profile questionnaire. Disabil Rehabil. 2019:1-5. https://doi.org/10.1080/09638288.2019.16539 $\underline{99}$ 
8. World Health Organization (WHO). Health promotion glossary [Internet]. Geneva: WHO; 1998. Disponível em: https://www.who. int/healthpromotion/about/HPR\%20Glossary\%201998.pdf?ua=1

9. Oliveira AP, Gomes LF, Casarin ST, Siqueira HCH. O viver do portador chagásico crônico: possibilidades de ações do enfermeiro para uma vida saudável. Rev Gaúcha Enferm. 2010;31(3):491-8. https://doi.org/10.1590/S198314472010000300012

10. Añes CRR, Reis RS, Petroski EL. Versão Brasileira do Questionário "Estilo de Vida Fantástico": Tradução e Validação para adultos jovens. Arq Bras Cardiol. 2008;91(2):92-8. https://doi. org/10.1590/S0066-782X2008001400006

11. Wilson MCD, Ciliska D. Lifestyle assessment: development and use of the FANTASTIC checklist. Can Fam Physician [Internet].1984;30:1527-32. Disponível em: https://www.ncbi.nlm. nih.gov/pmc/articles/PMC2153572/

12. Craig $C L$, Marshall $A L$, Sjostrom $M$, Bauman $A E$, Booth $M L$, Ainsworth $B E$, et al. International Physical Activity Questionnaire: 12-Country Reliability an Validity. Med Sci Sports Exerc. 2003;35(8):1381-95. https://doi.org/10.1249/01. $\underline{\text { mss.0000078924.61453.fb }}$

13. International Physical Activity Questionnaire. Guideline for data processing and analysis of the International Physical Activity Questionnaire (IPAQ) - Short and Long Forms [Internet]. 2005. Disponível em: https://www.researchgate.net/file.PostFileLoader $\cdot$ html?id=5641f4c36143250eac8b45b7\&assetKey=AS\% 3A294237418606593\%401447163075131

14. Braga JCV, Reis F, Aras RJ, Dantas N, Bitencourt A, Neves FS, et al. Is Chagas cardiomyopathy an independente risk fator for patients with heart failure?. Int J Cardiol. 2008;126(2):276-8. https://doi.org/10.1016/j.ijcard.2007.01.097

15. Barbosa AP, Cardinalli-Neto A, Otaviano AP, Rocha BF, Bestetti RB. Comparação do desfecho entre a Cardiopatia chagásica e a miocardiopatia dilatada idiopática. Arq Bras Cardiol. 2011;97(6):517-25. https://doi.org/10.1590/S0066$\underline{782 \times 2011005000112}$

16. Moctezuma RR, Carmona JML, Miranda CM, Santiago JLH, Bermúdez MM,. Validez y consistencia del instrumento Fantastic para medir estilo de vida en diabéticos. Rev Med Inst Mex Seguro Soc [Internet]. 2003;41(3):211-20. Disponível em: https://www. medigraphic.com/cgi-bin/new/resumen.cgi?IDARTICULO=2488

\section{abstract\&pid=S0120-53072011000300016}

18. Carmona-Lopez JM, Rodriguez-Moctezuma R, MunguíaMiranda C, Hernández-Santiago JL, Torre EC. Validez y fiabilidade del instrumento FANTASTIC para medir el estilo de vida em pacientes mexicanos com hipertensión arterial. Aten Primária. 2000;26(8):542-9. https://doi.org/10.1016/S0212-6567(00)78719-1

19. Bornhausen A, Kessler RMG, Gasperin SI. Qualidade subjetiva do sono em cardiopatas isquêmicos crônicos. Insuf Card [Internet]. 2018;13(3):110-7. Disponível em: https://pesquisa. bvsalud.org/portal/resource/pt/biblio-914519?src=similardocs

20. Edmealem A, Degu G, Haile D, Gedfew M, Bewket B, Andualem A. Sleep Quality and Associated Factors among Diabetes, Hypertension, and Heart Failure Patients at Debre Markos Referral Hospital, Northwest Ethiopia. Sleep Disord. 2020; 2020:6125845. https://doi.org/10.1155/2020/6125845

21. Jackson $Y$, Castillo $S$, Hammond $P$, Besson M, Brawand-Bron $A$, Urzola D, et al. Metabolic, mental health, behavioural and socioeconomic characteristics of migrants with Chagas disease in a non-endemic country. Trop Med Int Health. 2012;17(5):595-603. https://doi.org/10.1111/j.1365-3156.2012.02965.x

22. Mediano MFF, Leifer ES, Cooper LS, Keteyian SJ, Kraus WE, Mentz RJ, et al. Influence of Baseline Physical Activity Level on Exercise Training Response and Clinical Outcomes in Heart Failure: The HF-ACTION Trial. JACC Heart Fail. 2018;6(12):1011-9. https://doi.org/10.1016/j.jchf.2018.09.012

23. Costa HS, Lima MMO, Lage SM, Costa FSM, Figueiredo PHS, Rocha MOC. Six-minute walk test and incremental shuttle walk test in the evaluation of functional capacity in Chagas heart disease. J Exerc Rehabil. 2018;14(5):844-50. https://doi. org/10.12965/jer.1836326.163

24. Serafim TS, Santos EJ, Pierin MG. Influência do conhecimento sobre o estilo de vida saudável no controle de pessoas hipertensas. Acta Paul Enferm. 2010;23(5):658-64. https://doi. org/10.1590/S0103-21002010000500012

25. Urzúa AM. Calidad de vida y factores biopsicosociales em patologias médicas crónicas. Ter Psicol. 2008;26(2):207-14. http:// dx.doi.org/10.4067/S0718-48082008000200007

17. Araújo EPP, Souza MHN, Guimarães RM, Pavani GF, Correa HFS, Carvalho PM, et al. Estilo de vida de pacientes hipertensos atendidos com a Estratégia de Saúde Familiar. Invest Educ Enferm [Internet]. 2011;29(3):467-76. Disponível em: http://www.scielo.org.co/scielo.php?script=sci 